\title{
A SYSTEM OF WINE BLENDING BASED ON NEURAL NETWORK
}

\author{
Jianping Ren, Zhimin li \\ Department of Mechanical Engineering, North University of China, China; Email: \\ renip@nuc.edu.cn.
}

\begin{abstract}
According to the investigation of some liquor company, this paper built a physics and chemistry information database of wine bodies and a management information system, and achieved wine auto-blending through some training to physics and chemistry information of wine bodies by exercising BP network. At the same time, a satisfactory obligatory condition (the best feeling, the balance harmony of a variety of slight ingredient) was produced by making use of the linear programming, and the wine auto-blending was achieved.
\end{abstract}

Key words: wine bodies, BP network, wine auto-blending, linear-programming, Computer-aided wine blending.

\section{INTRODUCTION}

In practical working course, the quality of liquor is not stability because the quality of wine blending totally depends on the personal feeling of the person who is very knowledgeable about liquor (gustation and smell), and yet the personal ability has some ambiguity, and is subject to being effected by outside factors. For example, the person who is very knowledgeable sometimes feel good about the liquor, but its result of the chemical examination is not passed the standards of the state; or its result of the chemical examination is passed the standards of the state, but the person who is very knowledgeable feel not good about the liquor. Now the key problem should be solved at once that how to rebuild the traditional process of making-liquor and the experiential techniques of making-liquor to managing by computers and adopting mathematical method and neural network algorithm.

Please use the following format when citing this chapter:

Ren, Jianping, li, Zhimin, 2006, in International Federation for Information Processing (IFIP), Volume 207, Knowledge Enterprise: Intelligent Strategies In Product Design, Manufacturing, and Management, eds. K. Wang, Kovacs G., Wozny M., Fang M., (Boston: Springer), pp. 604-609. 


\section{THE ENSEMBLE PROJECT DESIGN OF THE SYSTEM}

According to the practical and process analysis of some liquor company, we fixed on the process of making-liquor (is shown as Figure 1). Management information system module of wine bodies is shown as Figure 2. The managing process of system work is shown as Figure 3 .

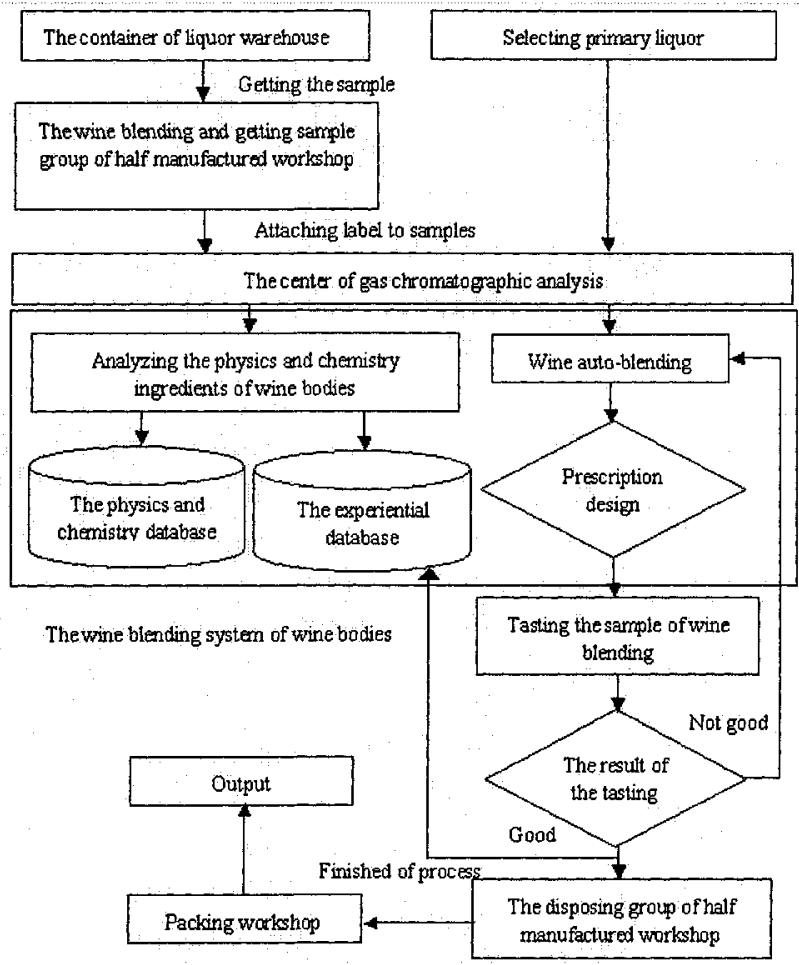

Figure 1. The process of making-liquor.

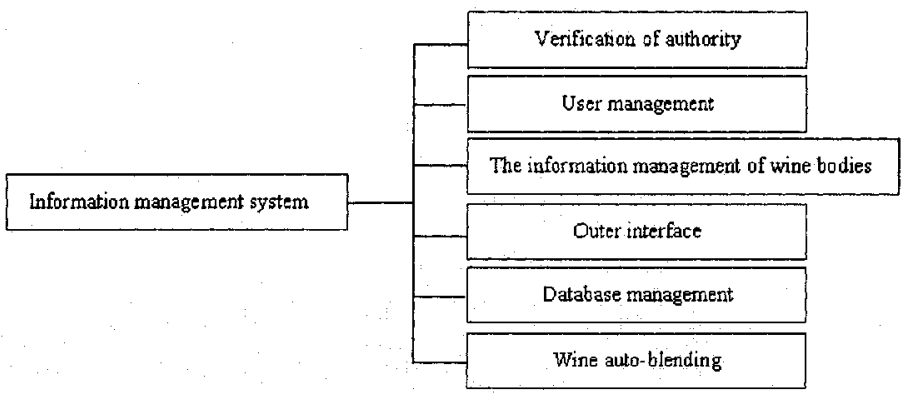

Figure 2. The management information system module of wine bodies. 


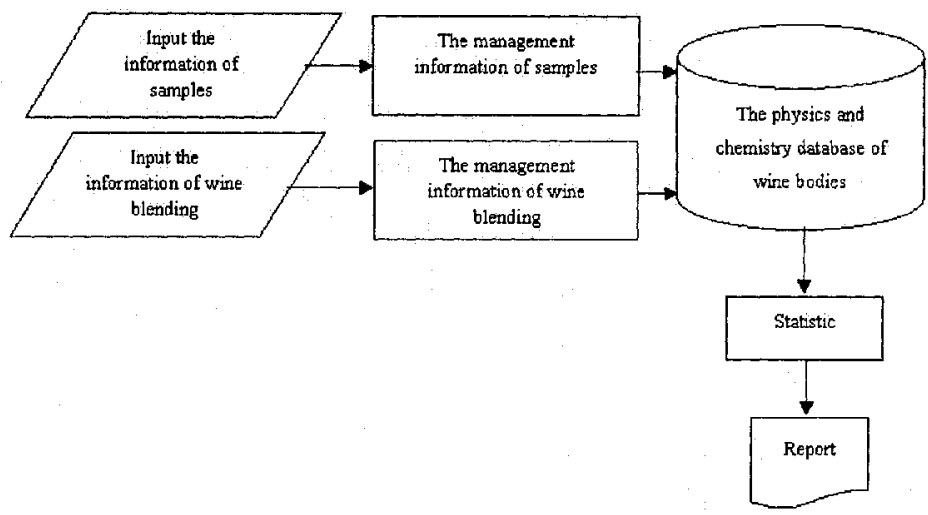

Figure 3. The managing process of system.

\section{THE IMPLEMENT OF WINE AUTO-BLENDING SUB MODULE}

Wine auto-blending was reformed tradition techniques to that: experimenters get small sample of different level liquor to test, and input the result into the system. Then the system automatically fills a prescription in accordance with the standards of the state, and its prescription adopts linear programming algorithm. For this reason, the wine blending will be with the standards of the state. In the end, the person who is very knowledgeable tastes the wine blending and adjusts blending parameters in accordance with the tasted feeling. So the whole process was under the standards, and it could improve the speed and the quality of the wine blending.

The designed user interface sub module of wine auto-blending is shown as Figure 4. Firstly, the range of the liquor index is input into the system, then it designs the suitable arithmetic to calculate the best method of mixing in terms of the range and information of the liquor. The linear programming and the object programming are important branches of the operational research, and it was studied and applied in earlier time and developed correspondingly mature. Introducing linear programming on the wine automixing sub module, we could make the wine blending more pertinence, veracity, maneuverability, and reducing the fluctuation of the quality.

The designed user interface sub module of wine auto-blending is shown as Figure 4. Firstly, the range of the liquor index is input into the system, then it designs the suitable arithmetic to calculate the best method of mixing 
in terms of the range and information of the liquor. The linear programming and the object programming are important branches of the operational research, and it was studied and applied in earlier time and developed correspondingly mature. Introducing linear programming on the wine automixing sub module, we could make the wine blending more pertinence, veracity, maneuverability, and reducing the fluctuation of the quality.

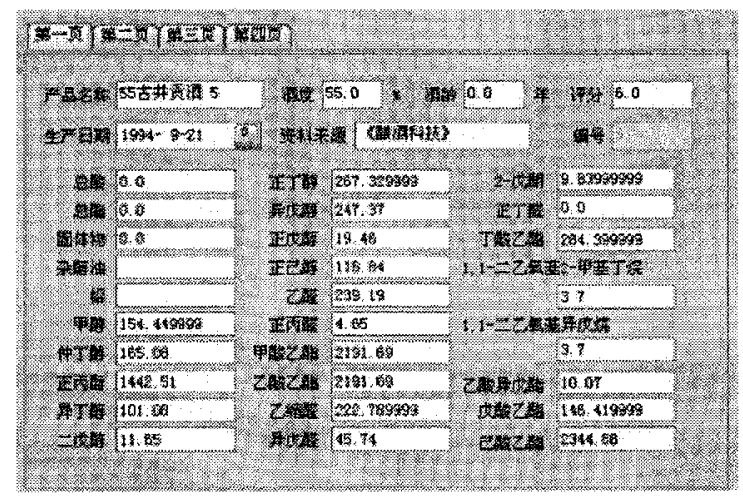

Figure 4. The input interface of physics and chemistry information of wine bodies.

When the wine blending, the indexes of physics and chemistry ingredients principally rest on the standard of the state and the corporations, and are compatible with the demand of foodstuff security and taste. The information of the physics and chemistry ingredients is dredged up by sub module of wine bodies, and data mining is good to test and verify if the standards of corporations are maturity, and could improve the product quality.

\section{THE IMPLEMENT OF ANALYZING PHYSICS AND CHEMISTRY INGREDIENTS OF WINE BODIES}

The sub module of analyzing physics and chemistry ingredients of wine bodies is established on the physics and chemistry indexes of wine bodies offered by a liquor manufactory. Except for the solids and the plumbum, 120 physics and chemistry ingredients of every wine body are all organic. More than ten of these ingredients are skeleton ingredients. That is what is known as important to the quality of liquor; the rest are not skeleton ingredients, and their effect is unknown too. The person who is very knowledgeable marks all samples of liquor. "The analysis of physics and chemistry ingredients of 
wine bodies" should get below knowledge: (1) the relation of physics and chemistry ingredients and mark; (2) the weighting of physics and chemistry ingredient in the network. At last, this module could mark for the sample of new type liquor.

There are three steps in the analyzing process. Firstly, with the liquor samples offered by users, we will design the database management system and provide some functions (including adding, deleting, changing, searching) for the information, in order to insure the validity of the data and the robustness of the program. Besides little additive information, there are about 120 floating-point types fields whose veracity decides the success. After building the winy sample information database, dactylogram atlas of the different liquor samples should be established to figure physics and chemistry information; secondly, we will design the interface which pick DBMS up MATLAB, and then input the physics and chemistry data of liquor samples to MATLAB to support the next step calculation; Lastly in the MATLAB, we will design proper neural network algorithm by making use of the toolbox of neural network to train the liquor samples data and dredge up the connotative information among them.

\section{DRAWING THE DACTYLOGRAM OF LIQUOR}

Just as everyone has his dactylogram and the dactylogram of everyone has its peculiarity, every kind of liquor sample has difference in its peculiarity and principal ingredient. With the help of computes and modem analyzing technology, the liquor sample peculiarity and its effective ingredients could be depicted in the way of atlas to make every kind of liquor has a standard atlas as the dactylogram of human. This is the dactylogram atlas of liquor.

When establishing the dactylogram atlas, we will firstly chose the typical sample to analyze, then draw the graph with different peak value in terms of different physics and chemistry ingredients of the sample. Then this atlas is the dactylogram atlas of this sample. The dactylogram atlas of Fenjiu-flavor liquor (A) and compared liquor sample (B) is shown as Figure 5.

When the quality of new produced liquor is tested, we could draw its physics and chemistry ingredients into dactylogram atlas, and analyze its Gas Chromatographic method. Then, compare it to the standard atlas in the database. For different the quality of liquor, we can find the factors that affect the quality by contrasting the dactylogram atlas. We also could introduce some mathematical tools, such as the cluster analysis algorithm, and as the assistance to estimate the quality. 


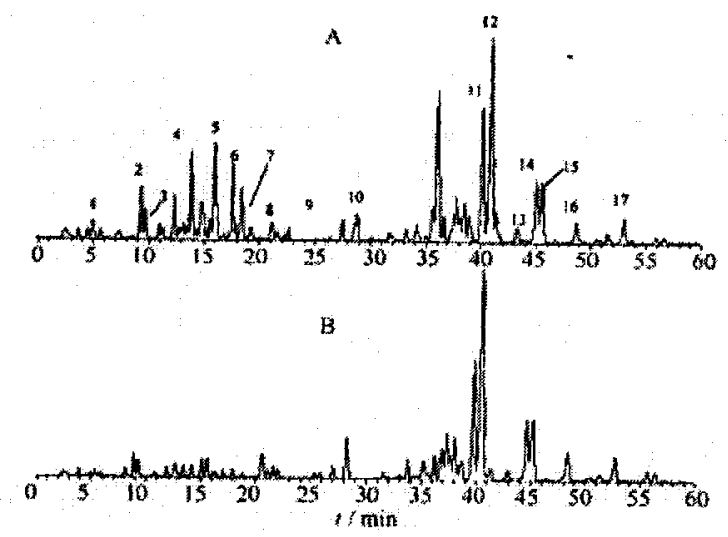

Figure 5. The dactylogram atlas of Fenjiu-flavor liquor (A) and compared liquor sample (B).

\section{CONCLUSION}

According to some liquor company, this paper was making use of $\mathrm{VC}++$ and SQL Server, and have designed a physics and chemistry information database of wine bodies and the management information system, and then has automatically marked for the quality of liquor from the information of physics and chemistry analysis. A satisfactory obligatory condition (the best feeling, the balance harmony of a variety of slight ingredient) was researched by making use of the linear layout, and achieved the wine autoblending.

\section{REFERENCES}

1. Zhai Xulong. The research and exploitation of characteristic ingredients dactylogram atlas system on Fen-flavor Liquor with Daqu. Project application report. 2004.8

2. Huang Hanying\&Wei Mingxin\&Yao Jicheng. The application of linear programming method in the technology of wine blending. Journal of Huazhong Agricultured University, 2001(4): 400-403. 